Laser Chem., 1999, Vol. 19, pp. 105-108 Reprints available directly from the publisher Photocopying permitted by license only
(C) 1999 OPA (Overseas Publishers Association) N.V. Published by license under the Harwood Academic Publishers imprint, part of The Gordon and Breach Publishing Group.

Printed in India.

\title{
THEORETICAL SIMULATION OF THE ZEKE SPECTRA OF NAPHTHALENE FROM SINGLE VIBRONIC LEVELS OF $S_{1}$
}

\author{
FABRIZIA NEGRI ${ }^{\mathrm{a}, *}$ and MAREK Z. ZGIERSKI ${ }^{\mathrm{b}}$ \\ a Dipartimento di Chimica "G. Ciamician", Università di Bologna, \\ Via F. Selmi, 240126 Bologna, Italy; \\ ${ }^{\mathrm{b}}$ Steacie Institute for Molecular Sciences, \\ National Research Council of Canada, Ottawa, K1A 0R6 Canada
}

(Received 7 April 1997)

We present the simulations and analysis of the two-color ZEKE spectra of naphthalene, performed with the help of quantum chemical calculations of molecular parameters followed by the modelling of vibronic intensities. $A b$ initio and semi-empirical calculations were carried out to obtain molecular structures of neutral and ionic naphthalene, and vibronic perturbations that couple the electronic states. It is shown that the intensities, simulated with a model based on the perturbative expansion of vibronic states, nicely reproduce the observed spectra and contribute to reassign some of the ground state frequencies of naphthalene cation.

Keywords: ZEKE spectra; simulations; semi-empirical calculations; ab initio calculations; vibronic interactions

The measurement of zero kinetic energy (ZEKE) photoelectron spectra depends on the long life-time of the highly excited Rydberg states of molecules [1] which lie just below the threshold for photoionization. Since Rydberg electrons interact only marginally with the core of the molecule, the vibronic structure of the ZEKE spectra generally reflects the geometry change between the neutral species and the ion. In this sense, the Franck-Condon (FC) mechanism usually suffices to explain the observed vibronic intensities.

\footnotetext{
${ }^{*}$ Corresponding author.
} 
The two-color ZEKE spectra of naphthalene recently measured by Cockett et al. [2] by pumping several single vibronic levels of $S_{1}$, show clear deviations from the $\Delta v=0$ propensity rule usually observed for nontotally symmetric modes (NTS). This indicates that other mechanisms are operating, and suggests the presence of non-negligible vibronic coupling effects.

We have recently analyzed the vibronic structure of the single vibronic level (SVL) fluorescence spectra of naphthalene [3] by performing simulations based on the perturbative expansion of vibronic states. The agreement between observed and simulated spectra was very satisfactory and here we have extended the model of Ref. [3] to simulate the vibronic structure of ZEKE spectra of naphthalene. A schematic representation of the states involved in the model is presented in Figure 1, where $V\left(b_{1 g}\right)$ are the vibronic perturbations that couple $S_{1}$ and $D_{0}$ to higher excited states. As shown in Figure 1, the mechanism activates the FC forbidden $S_{1}\left(0, B_{3 u}\right) \rightarrow D_{0}\left(1, B_{1 u}\right)$ vibronic transition by-borrowing intensity from the $S_{2} \rightarrow D_{0}$ and the $S_{1} \rightarrow D_{1}$ transitions. The vibronic perturbations that couple the $S_{1}$ and $D_{0}$ states to low-lying excited states were evaluated with the $\mathrm{CNDO} / \mathrm{S}$ hamiltonian augmented with Configur-

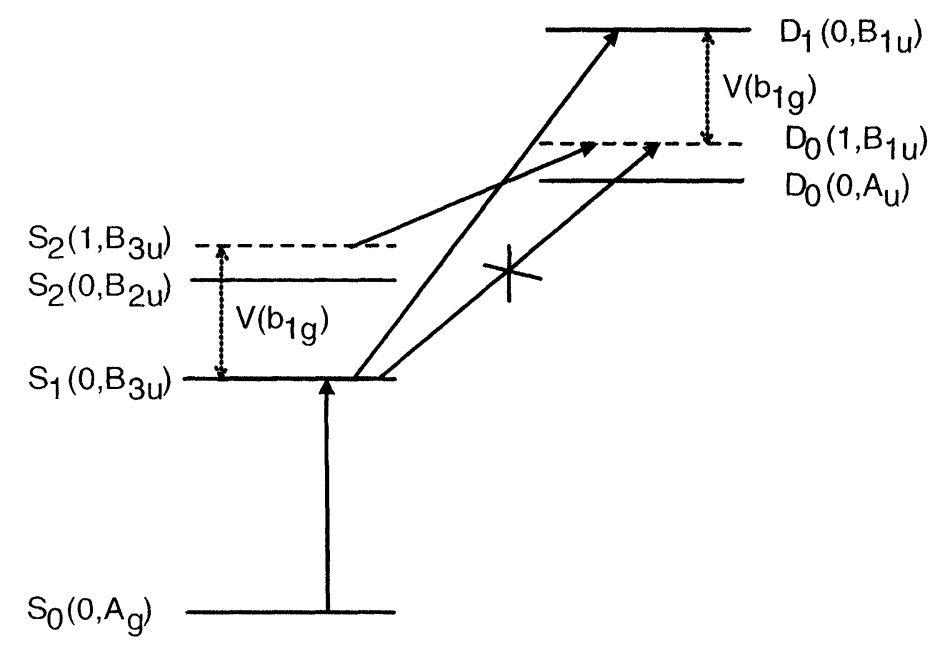

FIGURE 1 Schematic representation of the model employed to simulate the vibronic activity in the ZEKE spectra of naphthalene. 
ation Interaction calculations [5], while the remaining molecular parameters required for the simulations were computed at the ab initio level of theory by using the Gaussian 92 suite of programs [4].

In Figure 2 we present the simulations of the ZEKE spectra originated from the $0_{0},(8 b)_{1},(9 a)_{1}$ and $(8 a)_{1}$ vibronic levels of $S_{1}$. The

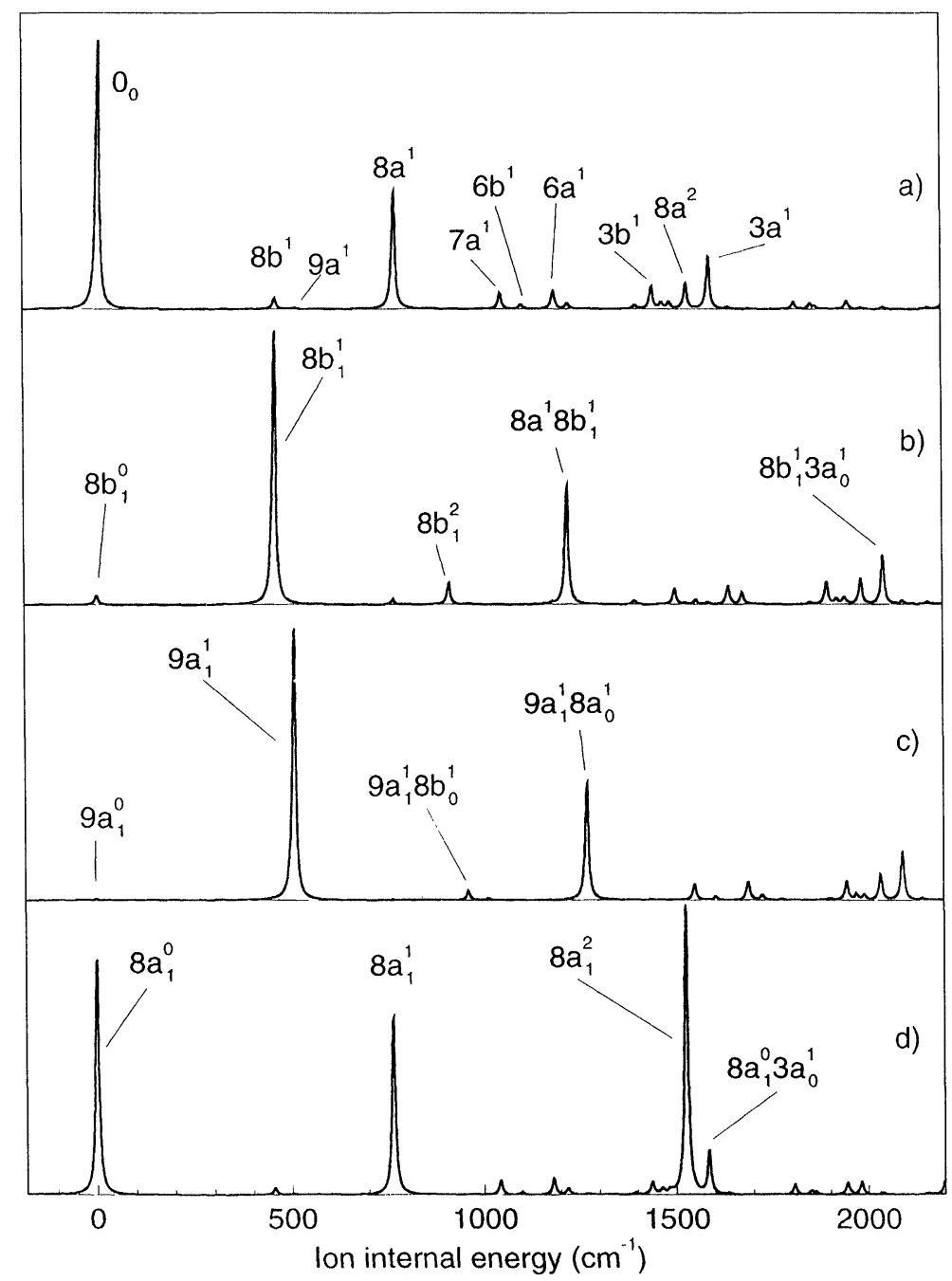

FIGURE 2 Simulations of the ZEKE spectra of naphthalene: (a) from the $0_{0}$ level of $S_{1}$; (b) from the $(8 b)_{1}$ level of $S_{1}$; (c) from the $(9 a)_{1}$ level of $S_{1}$; (d) from the $(8 a)_{1}$ level of $S_{1}$. 
calculated spectra reproduce nicely the main features of the observed spectra (see Fig. 3 of Ref. [2]) and point to several reassignments of the fundamentals of naphthalene cation, some of which are indicated in the figure. Inspection of the computed contributions to vibronic intensities indicates that the couplings between doublet states are mainly responsible for the observed activity of NTS modes. The latter couplings are indeed one order of magnitude larger than those between singlet states. In conclusion we have demonstrated that quantumchemical calculations combined with the modelling of vibronic intensities can be employed to analyze the structure of ZEKE spectra even when the Herzberg Teller mechanism of intensity borrowing becomes an important source of intensity.

\section{References}

[1] Müller-Dethlefs, K. and Schlag, E. W. (1991). Annu. Rev. Phys. Chem., 42, 109.

[2] Cockett, M. C. R., Ozeki, H., Okuyama, K. and Kimura, K. (1993). J. Chem. Phys., 98, 7634 .

[3] Negri, F. and Zgierski, M. Z. (1996). J. Chem. Phys., 104, 3486.

[4] Gaussian 92/DFT, Revision G.2, Frisch, M. J., Trucks, G. W., Schlegel, H. B., Gill, P. M. W., Johnson, B. G., Wong, M. W., Foresman, J. B., Robb, M. A., HeadGordon, M., Replogle, E. S., Gomperts, R., Andres, J. L., Raghavachari, K., Binkley, J. S., Gonzalez, C., Martin, R. L., Fox, D. J., Defrees, D. J., Baker, J., Stewart, J. J. P. and Pople, J. A. (1993). Gaussian, Inc., Pittsburgh PA, 1993.

[5] Del Bene, J. and Jaffe, H. H. (1968). J. Chem. Phys., 48, 1807. 